\title{
H-389 0, HÍBRIDO TRILINEAL DE MAÍZ DE GRANO BLANCO OLEOSO ADAPTADO A REGIONES SUBTROPICALES DE MÉXICO
}

\section{H-389 0, A WHITE KERNEL THREE-WAY MAIZE HYBRID OF HIGH OIL CONTENT ADAPTED TO SUBTROPICAL REGIONS OF MEXICO}

\author{
Ricardo E. Preciado-Ortiz ${ }^{\star}$, Arturo D. Terrón-Ibarra', Ma. Gricelda Vázquez-Carrillo², \\ Noel O. Gómez-Montiel ${ }^{3}$, Dolores Briones-Reyes ${ }^{4}$ y Alfonso Peña-Ramos ${ }^{4}$
}

\begin{abstract}
${ }^{1}$ Instituto Nacional de Investigaciones Forestales, Agrícolas y Pecuarias (INIFAP), Campo Experimental Bajío, Celaya, Guanajuato. México. ${ }^{2}$ INIFAP, Campo Experimental Valle de México, Texcoco, Estado de México, México. ${ }^{3}$ INIFAP, Campo Experimental Iguala, Iguala, Guerrero, México. INIFAP, Campo Experimental Pabellón, Pabellón de Arteaga, Aguascalientes, México.
\end{abstract}

*Autor para correspondencia (preciado.ernesto@inifap.gob.mx; repreciado@yahoo.com)

En las regiones subtropicales de México, el mejoramiento genético del maíz (Zea mays L.) se ha enfocado tradicionalmente hacia la obtención de híbridos de alto rendimiento; sin embargo, las necesidades nutrimentales e industriales han reorientado los programas de mejoramiento para incluir aspectos de calidad nutricional e industrial del grano (Ortiz-Monasterio et al., 2007), donde el maíz con alto contenido de aceite (ACA) es de gran importancia para las industrias aceitera y de alimentos balanceados. Un maíz ACA contiene más del $6 \%$ de aceite, en comparación con el maíz normal que tiene entre 2.0 y $5.0 \%$. El maíz ACA tiene valor nutricional adicional por su energía metabolizable, con un efecto significativo en raciones para animales, y puede sustituir grasas, harina de soya o aminoácidos esenciales sintéticos, lo que mejora la dieta (Alexander, 1988; Lambert, 2000). Una serie de estudios revisados por Lambert (2000) con cerdos, aves, ovejas y vacas lecheras, alimentados con raciones de maíz ACA mostraron en ellos mayor crecimiento, eficiencia alimenticia y calidad de sus productos.

El híbrido H-389 O, de grano blanco ACA (oleoso), adaptado a regiones subtropicales de México, es una alternativa para impactar la producción agrícola, pecuaria e industrial en la cadena productiva del maíz. El H-389 O es un híbrido trilineal, cuyo progenitor femenino es la cruza simple (LBDHBO4 $\times$ LBDHBO5) formada con líneas doble haploides (LDH) derivadas de la Población Blanca del Noroeste (PBN), y el progenitor masculino (LBDHBO2) es una LDH derivada de la Población Blanca del Bajío (PBB) (Cuadro 1). La Población Blanca del Noroeste incluye híbridos y líneas con excelente adaptación al noroeste de México, presenta resistencia a la roya de la hoja (Puccinia polysora) y excelente calidad de tallo. La Población Blanca del Bajío proviene de la variedad $\vee$ 385, derivada de la Población de Amplia Base Genética Tardía; involucra germoplasma subtropical, básico, experimental, colectas e introducciones (Ortega-Corona et al., 2015).
El proceso de selección para ACA en PBN y PBB se inició a partir de 2004 bajo un esquema de selección recurrente de familias de medios hermanos. En campo se seleccionaron las cuatro mejores mazorcas de cada familia provenientes de plantas sanas, con competencia completa, sin acame y con altura de planta y mazorca agronómicamente aceptables. En laboratorio, entre las 200 familias seleccionadas en campo se seleccionaron las 50 familias con ACA (presión de selección de 25 \%) mediante un espectrofotómetro de rayos infrarrojos cercanos (NIRS, InfraTec 1241, Plano, Texas, USA), que analiza en conjunto todas las semillas de cada mazorca. Después, se realizó selección dentro de las 50 familias superiores a través del NIRS (InfraTec 1255, Plano, Texas, USA), lo que permitió identificar las semillas individuales con mayor contenido de aceite provenientes de cada mazorca. La respuesta a la selección de estas poblaciones fue reportada por PreciadoOrtiz et al. (2013) y por Ortega-Corona et al. (2015).

Las LDH progenitoras del H-389 O fueron derivadas del octavo y séptimo ciclo de selección para ACA en PBN y PBB. La derivación de las LDH se inició en el año 2011 con los siguientes pasos: 1) inducción de haploidía a través de la cruza de PBN y de PBB con polen del inductor RWS $\times$ UH400, 2) identificación de semilla haploide mediante el marcador Navajo R1-nj, 3) duplicación de los haploides con colchicina como inhibidor mitótico y 4) autofecundación de plantas duplicadas para incrementar la semilla de las nuevas LDH (Prasanna, 2012). El incremento, selección y cruzamientos de LDH se realizó en el Campo Experimental Bajío del Instituto Nacional de Investigaciones Forestales, Agrícolas y Pecuarias (INIFAP) a partir del ciclo primaveraverano de 2012.

En el año 2018, el híbrido H-389 o fue registrado ante el Servicio Nacional de Inspección y Certificación de Semillas (SNICS) con la clave: MAZ-1855-090318 en el Catálogo Nacional de Variedades Vegetales (CNVV) y el Título de 
Obtentor con número de registro 2042. El H-389 O es de alto potencial de rendimiento, ciclo intermedio, porte alto de planta, tolerancia al acame, mazorca muy larga, con 14 a 16 hileras, ligeramente en espiral, sana, con buena cobertura, grano dentado, color blanco cremoso y corona de forma hendida (Figura 1), la espiga es muy larga, con presencia intermedia de ramas laterales. El progenitor femenino es de alto potencial de rendimiento, ciclo intermedio, porte de planta intermedio, mazorca intermedia con 16 a 18 hileras, grano dentado, color blanco cremoso y corona de forma hendida. El progenitor masculino, es de ciclo intermedio, porte bajo, mazorca mediana de 14 a 16 hileras, grano semi-cristalino, blanco cremoso, corona de forma convexa, espiga mediana, compacta, y bajo número de ramas principales.

Cuadro 1. Denominación, genealogía y obtentor de los progenitores del híbrido trilineal de maíz $\mathrm{H}-3890$ de grano blanco y alto contenido de aceite.

\begin{tabular}{llll}
\hline & Parental A & Parental B & Parental C \\
\hline Denominación & LBDHBO 4 & LBDHBO 5 & LBDHBO 2 \\
Genealogía & PBN DH 5 & PBN DH 16 & PBB DH 183 \\
Obtentor & INIFAP & INIFAP & INIFAP \\
\hline
\end{tabular}

El híbrido H-389 O fue evaluado durante los años 2014, 2015 y 2016 en diversos ambientes de la región subtropical de México, con un promedio de rendimiento de grano de $12.7 \mathrm{t} \mathrm{ha}^{-1}$ con valores de 8.3 a $17.8 \mathrm{t} \mathrm{ha}^{-1}$, con rangos de floración masculina de 69 a 81 y femenina de 70 a 83 d; alturas de planta de 158 a 330 y de mazorca de 83 a $180 \mathrm{~cm}$. En el Cuadro 2, se presenta el promedio de ocho ambientes para rendimiento de grano y características físico-químicas del grano del híbrido H-389 O, en comparación con el testigo normal H-377. El híbrido H-389 $\mathrm{O}$ tuvo mayor rendimiento y contenido de aceite que el testigo; pero su grano fue menos duro, más pequeño y con menor contenido de almidón. El grano de ambos híbridos fue similar en peso hectolítrico y contenido de proteína. Por sus características, el H-389 O puede ser destinado a la industria de la masa y la tortilla, ya que cumple con los parámetros demandados en la Norma NMX-034 (SE, 2002; Vázquez-Carrillo et al., 2018), así como impactar en diversos sectores de la cadena productiva del maíz. La semilla básica del progenitor masculino (LBDHBO2) y registrada del progenitor femenino (LBDHBO4 $\times$ LBDHBO5) está disponible, previa solicitud al INIFAP, para los interesados en la producción de semilla certificada del H-389 O.

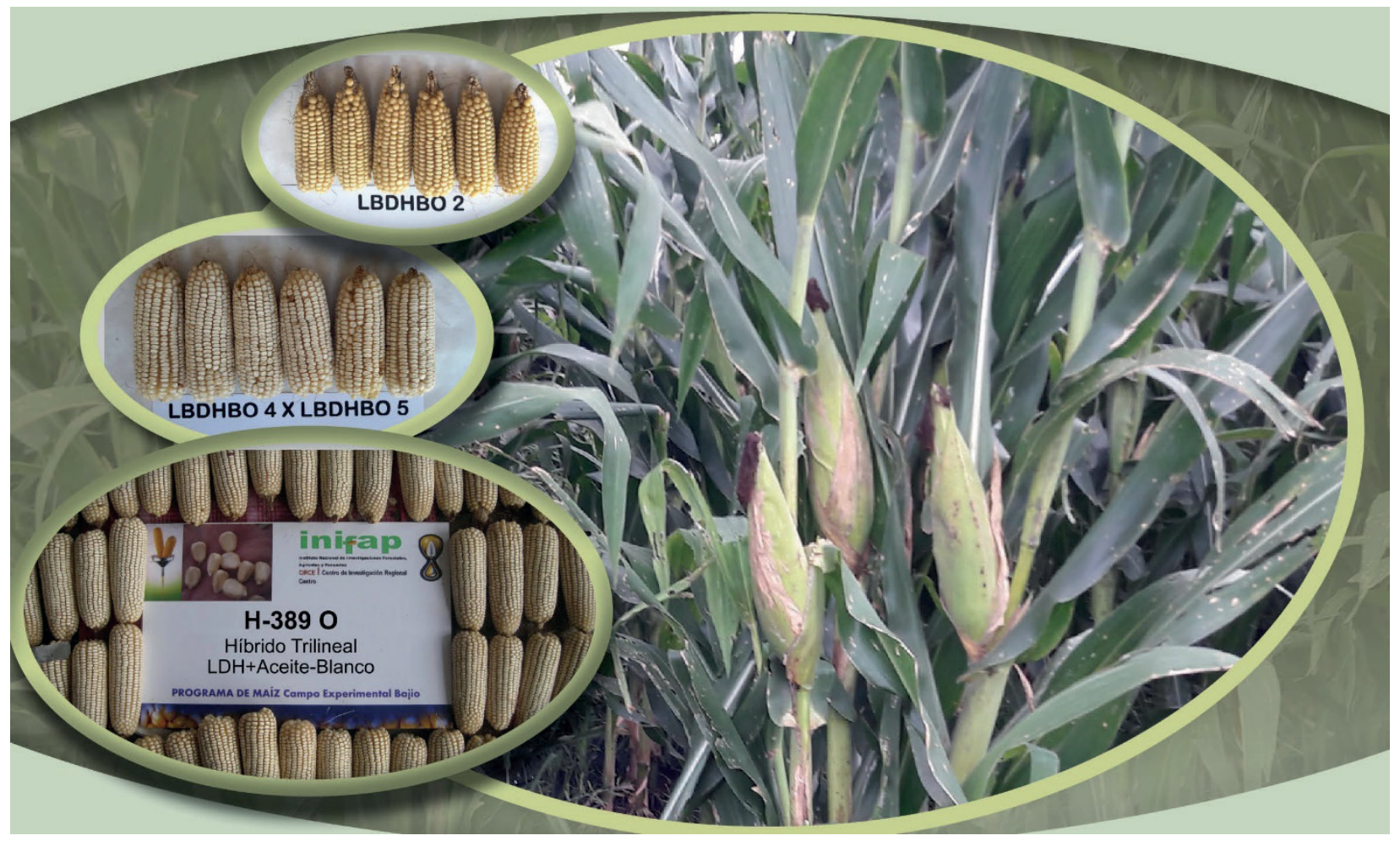

Figura 1. Carcaterísticas morfológicas del híbrido H-389 0, de grano blanco oleoso. 
Cuadro 2. Medias del rendimiento y calidad de grano del híbrido trilineal de maíz H-389 0 de grano blanco y alto contenido de aceite en ocho ambientes comparado con el híbrido $\mathrm{H} 377$.

\begin{tabular}{lccccccc}
\hline Genotipo & $\begin{array}{c}\text { Rendimiento } \\
\left(\mathrm{t} \mathrm{ha} \mathrm{-}^{-1}\right)\end{array}$ & $\begin{array}{c}\text { Índice de } \\
\text { Flotación (\%) }\end{array}$ & $\begin{array}{c}\text { Peso de } 100 \\
\text { granos }(\mathrm{g})\end{array}$ & $\begin{array}{c}\text { Peso hectolítrico } \\
\left(\mathrm{kg} \mathrm{hL}^{-1}\right)\end{array}$ & $\begin{array}{c}\text { Contenido de } \\
\text { aceite }(\%)\end{array}$ & $\begin{array}{c}\text { Contenido de } \\
\text { almidón (\%) }\end{array}$ & $\begin{array}{c}\text { Contenido de } \\
\text { proteínas }(\%)\end{array}$ \\
\hline $\mathrm{H}-389 \mathrm{O}$ & $12.07 \mathrm{a}$ & $23.06 \mathrm{a}$ & $29.30 \mathrm{~b}$ & $78.05 \mathrm{a}$ & $6.24 \mathrm{a}$ & $67.36 \mathrm{~b}$ & $9.77 \mathrm{a}$ \\
$\mathrm{H}-377$ & $10.05 \mathrm{~b}$ & $15.63 \mathrm{~b}$ & $35.23 \mathrm{a}$ & $78.51 \mathrm{a}$ & $5.07 \mathrm{~b}$ & $69.10 \mathrm{a}$ & $9.49 \mathrm{a}$ \\
$\mathrm{DMS}^{+}$ & 1.87 & 2.17 & 0.62 & 0.72 & 0.23 & 0.65 & 0.32 \\
\hline
\end{tabular}

${ }^{\dagger}$ Medias con letras iguales en las columnas no son estadísticamente diferentes (DMS, P $\leq 0.05$ ). Los ocho ambientes de evaluación fueron: Celaya, Gto. $20151^{\text {a }}$ fecha; Celaya, Gto. $20152^{\text {a }}$ fecha; Pabellón, Ags. 2015; Tarimbaro, Mich 2015; Celaya, Gto. $20161^{\text {a }}$ fecha; Celaya, Gto. $20162^{a}$ fecha; Tarimbaro, Mich 2016; y Juventino Rosas, Gto. 2016.

\section{BIBLIOGRAFÍA}

Alexander D. E. (1988) Breeding special nutritional and industrial types. In: Corn and Corn Improvement. G. F. Sprague and J. W. Dudley (eds.). Third edition. Agronomy Monograph No. 18. American Society of Agronomy, Crop Science Society of America, Soil Science Society of America. Madison, Wisconsin, USA. pp:869880

Lambert R. J. (2000) High-oil corn hybrids. In: Specialty Corns. Second edition. A. R. Hallauer (ed.). CRC Press. Boca Raton, Florida, USA. pp:131-154.

Ortega-Corona A., R. Picón-Rico, R. E. Preciado-Ortiz, A. D. Terrón-Ibarra, M. J. Guerrero-Herrera, S. García-Lara and S. O. Serna-Saldivar (2015) Selection response for oil content and agronomic performance in four subtropical maize populations. Maydica 60:1-8

Ortiz-Monasterio J. I., N. Palacios-Rojas, E. Meng, K. Pixley, R. Trethowan and R. J. Peña (2007) Enhancing the mineral and vitamin content of wheat and maize through plant breeding. Journal of Cereal Science 46:293-307, https://doi.org/10.1016/j.jcs.2007.06.005
Prasanna B. M. (2012) Doubled haploid (DH) technology in maize breeding: an overview. In: Doubled Haploid Technology in Maize Breeding: Theory and Practice. B. M. Prasanna, V. Chaikam and G. Mahuku (eds). CIMMYT. México, D. F. pp:1-8.

Preciado-Ortiz R. E., S. García-Lara, S. Ortiz-Islas, A. Ortega-Corona and S. 0. Serna-Saldivar (2013) Response of recurrent selection on yield, kernel oil content and fatty acid composition of subtropical maize populations. Field Crops Research 142:27-35, https:// doi.org/10.1016/j.fcr.2012.11.019

SE, Secretaría de Economía (2002) NMX-FF-034/1-SCFI-2002 Productos alimenticios no industrializados para consumo humano. Cereales. Parte I: Maíz blanco para proceso alcalino para tortillas de maíz y productos de maíz nixtamalizado. Especificaciones y métodos de prueba. Secretaría de Economía, México, D. F. 16 p.

Vázquez-Carrillo M. G., R. E. Preciado-Ortíz, D. Santiago-Ramos, N. PalaciosRojas, A. Terrón I. y A. Hernández-Calette (2018) Estabilidad del rendimiento y calidad de grano y tortilla de nuevos híbridos de maíz con valor agregado para el subtrópico de México. Revista Fitotecnia Mexicana 41:509-518. 
